\title{
Drop impact onto attached metallic meshes: liquid penetration and spreading
}

\author{
Cristina Boscariol $^{1, *}$, Sanjeev Chandra ${ }^{2}$, Dipak Sarker $^{3}$, Cyril Crua $^{1}$, Marco Marengo $^{1}$ \\ ${ }^{1}$ School of Computing Engineering and Mathematics, University of Brighton, United Kingdom \\ ${ }^{2}$ Department of Mechanical and Industrial Engineering, University of Toronto, Canada \\ ${ }^{3}$ School of Pharmacy and Biomolecular Science, University of Brighton, United Kingdom \\ *Corresponding author: c.boscariol@brighton.ac.uk
}

\begin{abstract}
The interaction between drops and porous matter has important applications in many fields such as painting, paper coating, design of textiles, filtration and therapeutic delivery, the latter can include also reconstructive surgery processes. Since the phenomenon of droplet impact onto a porous surface is particularly complex, a first step consists in analysing impacts on 2D structures, such as metallic porous layers. The present paper shows the case of drop impacts onto metallic meshes attached to a solid substrate. The pores are squared and not planar, due to the woven structure of the meshes: the dynamics of the flow is particulary complex, but it resembles more realistic cases. In analysing the impact of droplets of water, acetone and a mixture of glycerol and water on meshes with different pore sizes, three main outcomes were observed for both test cases: deposition, partial imbibition and penetration. Higher velocity impacts lead to droplet splashing followed by deposition, partial imbibition and penetration. A higher amount of liquid penetration is linked to a higher velocity impact, lower viscosity and a larger dimension of the pore size. A map of the regimes is proposed introducing two dimensionless numbers $\mathbf{M}$ and $\gamma$, that are functions of the Weber and Reynolds numbers and pore and wire sizes. Previous papers have not considered the role of the wire diameter. The two numbers allow a clear separation of the outcomes and a practical use of the results.
\end{abstract}

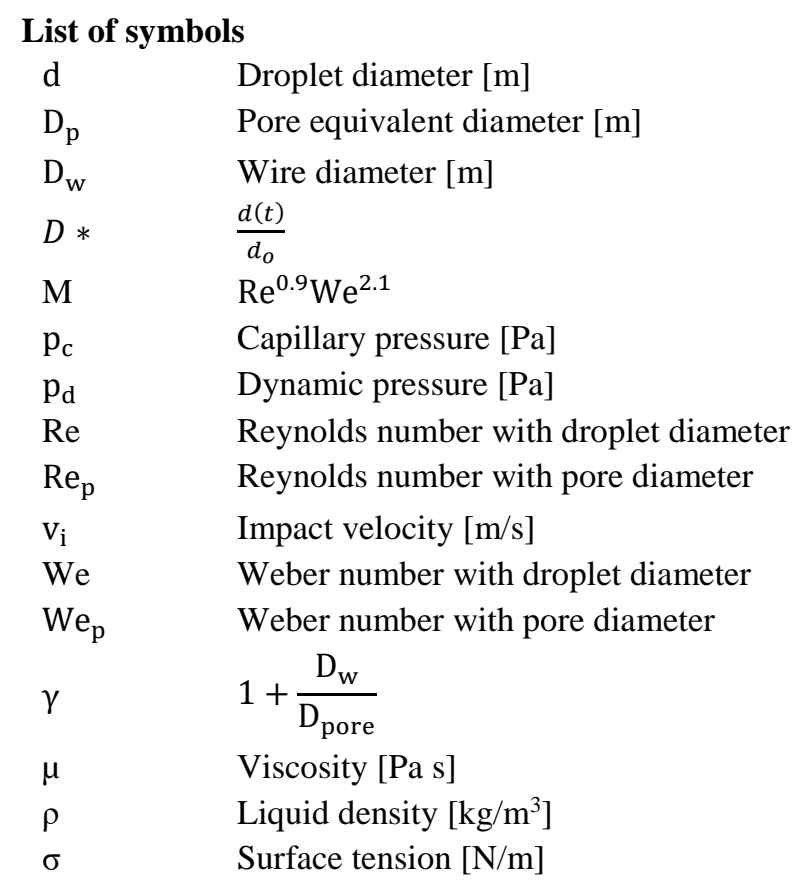




\section{Introduction}

Studies of liquid droplets impacting on solid surfaces are driven by their many applications in nature and industry. A typical phenomena in nature is that of rain droplets impacting on earth, stones or leaves, while industrial applications include spray/wall interactions in processes such as coating, cleaning, cooling and combustion (Josserand and Thoroddsen 2016). The behaviour of droplets impacting on smooth, flat surfaces has been extensively studied and is nowadays well understood. However, if the surface topology is complex, the outcome of a droplet collision is much harder to predict. Only a few studies have focussed on droplet interactions with complex surfaces, and the subject of droplet impact and penetration on a porous substrate is still poorly understood, even though it has important applications in many fields. The infiltration of rain drops and surface water into soil and the migration of oil in permeable porous media are both important subjects in environmental science (Yarin 2006). Agrochemicals are typically distributed on plants by spraying them onto leaf surfaces into which they penetrate (Bertola 2008). Porous surfaces find application in internal combustion engines as it is possible to enhance fuel vaporization by spraying fuel droplets on a porous coating on the piston crown (Weclas 2010). The quality of images produced by ink-jet printing depends on the penetration and spreading of ink droplets landing on paper (Kim and Lee 2014). Droplet impact dynamics can affect the result of cell printing (He et al. 2015). Suppression of fires by sprinkler systems involves droplets impinging on burning porous materials (Chandra and Avedisian 1992).

Since the understanding of the parameters that play the most important role in the spreading and penetration of a droplet landing on a porous surface is still incomplete, and further numerical and experimental investigations are required to quantify the imbibition of liquid into the porous surface and identify possible outcomes of droplet impacts, a number of experimental studies have been carried out on the subject.

Depending on its size and velocity, droplet spreading is affected by the porosity of the substrate and its evolution is modified by the surface structure. In order to understand the transport process on a macroscopic scale, it is necessary to have detailed information of processes taking place at both micro-and nano- length scales (Gambaryan-Roisman 2014). Porous media can be classified according to the amount of liquid that has penetrated into its pores: dry porous media is filled with air; saturated porous media is completely filled with liquid; and partially filled porous media contain a mixture of the two phases.

Roisman et al. (2015) developed a model describing impact of droplets on rough and porous surfaces and the threshold for splashing to occur. They proposed an experimental map showing that the two most significant parameters influencing transition from deposition to splashing are the Weber number and the ratio given by two geometrical characteristics linked to roughness (the average height of protruding peaks above roughness core profile and the mean width of a profile element). Sahu et al. (2015) analysed the impact of droplets of liquid suspensions onto porous filter membranes and concluded that penetration of liquid into porous medium is possible when the dynamic pressure is higher than the capillary pressure, and also when hydrodynamic focusing, which occurs when the drop diameter is much larger than the pore diameter, is observed. Clarke et al.(2002) combined a model linked to the relaxation of a sessile drop on an impermeable solid surface with an equation to describe the imbibition process on a porous surface. Kumar and Deshpande (2006) pointed out that the imbibition regime is influenced both by the material of the porous media and the properties of the liquid, and showed that increasing the drop size leads to a slower imbibition. Karapetsas et al. (2016) investigated droplet sliding on an inclined plane, considering both smooth and structured surfaces, and pointed out how dynamic hysteresis can be linked to the topography of the substrate. Similarly, Romdhani et al. (2014) analysed droplet impact on textiles, including woven cotton fabrics, and identified three different drop-spreading phases, that were influenced by fabric porosity. Kim and Lee (2014) studied the thermal and hydrodynamic behaviour of water drops impinging on heated porous surfaces and observed that increasing the size of the sintered pores reduced the extent of droplet spreading ratio and the permeation time. Yamamoto et al. (2016) investigated droplet impact on textured surface fabricated by aligning stainless-steel razor blades in parallel. They observed partial penetration of liquid into the gaps between blades when the impact Weber number was between 5 and 10 due to the collapse of the air cavity at the centre of the recoiling droplet. Hicks and Purvis (2017) compared the droplet impact onto a porous substrate with that of a rough impermeable surface and found that the trapped air-pocket was smaller for a porous surface as surface pores provided additional pathways for the gas to escape.

Lorenceau and Quéré (2003) valuated the impact of droplet on thin perforated plates and identified a critical speed above which the droplet was not captured entirely by the plate but passes through it. Delbos et al. (2010) investigated how an impacting drop can be forced into a capillary tube and observed a variety of regimes of droplet rebound or penetration for different combinations of impact velocity, tube radius and wetting condition. Liu and Wang (2016) 
described droplet impact on superhydrophobic surfaces covered with tapered posts. They presented a diagram illustrating three different impact regimes, using a geometrical dimensionless parameter given by the ratio of the product between the pore dimension and the height of the pillar divided by the spacing between each pillar. Xu et al. (2017) performed an investigation on impact of water droplets on mesh membranes that are used for gas-water or oilwater separation. They determined that there is a critical velocity for which a daughter droplet will be generated below the pore after impact, and pointed out that the drop impact on the membrane may result in a significant liquid compression, generating a water hammer pressure that enhances droplet penetratrion. The water hammer pressure was used also by Pittoni et al. (2014) to explain their results from a study of water droplets impacting on graphite substrate at low Weber number. In a similar study, Ryu et al. (2017) observed droplet water impact on meshes with submillimeter pores, focusing on the effect of varying surface wettability, hydrophobicity and superhydrophobicity. They observed that a higher impact velocity is required to allow penetration in the case of hydrophobic surfaces, whereas in the case of the super-hydrophobic meshes, penetration can occur at a lower velocity. They suggested that this difference was due to hydrodynamic focusing or to momentum transfer from the drop when it is about to bounce from the surface. Brunet et al. (2009) analysed droplet impact on hydrophobic microgrids and observed that above a threshold speed liquid penetrates through the grid forming microdroplets and proposed a method of producing monodisperse spray.

Zhang et al. (2018) analysed the spreading evolution of a droplet impacting both hydrophobic and hydrophilic textiles observing three different outcomes: capture, no penetration (a portion of the droplet penetrates the textile but is driven back to the top surface) and penetration (a fraction of the droplet permanently penetrates the textile). They pointed out that the outcome depends on a balance between the dynamic impact pressure and the capillary pressure, which are functions of pore size, liquid properties and droplet diameter.

A number of numerical models have been developed to simulate droplet impact on complex surfaces. Reis et al. (2004), presented a numerical model, based on the finite volume method, to analyse the dynamics of a liquid droplet impinging on a porous medium. Via enhancing and remarking the effects of surface tension and capillary forces they found good agreement with experimental data. Moqaddam et al. (2017), focused on the regime of bouncing on macro-textured superhydrophobic surfaces using the entropic lattice-Boltzman model for multiphase flows. Focusing on pancake bouncing phenomenon, characterised by a flattened rebound of the droplet, they accurately estimated the transformation of kinetic energy to surface energy and vice-versa. Choi et al. (2017) developed a sharp-interface LS method to analyse droplet impact and penetration into a porous medium. They demonstrated that the extent of droplet penetration depends on the initial droplet radius, impact velocity, and the porosity and wettability of the surface. Zhang et al. (2014) used molecular dynamics and a lattice-Boltzmann simulation technique to study the impact of microscopic liquid droplets on solid surfaces with varying surface characteristics. In considering droplet impact on a surface with a cross-shaped region of one wettability superimposed on a background with a different value, they demonstrated that the final drop shape is determined by the local rate of liquid spreading. Lee et al. (2016) presented a combined experimental and numerical analysis of the absorption of water droplets impacting porous stones, capturing the full absorption process of an impinging droplet. They observed that for a short time after the impact, before the absorption phase, the droplet spreads without any mass penetration into the substrate, due to the presence of an air layer between the droplet and the porous substrate.

Most of the studies of droplet impact on meshes or porous surfaces, focus on defining the velocity threshold criterion for which penetration occurs without considering the influence of different liquid physical properties or analysing the outcome for higher impact velocities for which a splash or a complete penetration of droplet below the surface may occur. This work allows predicting the outcome of droplet impact on mesh membranes at different time scale, considering liquid having different physical properties and meshes with a wide range of pores size.

The objective of this study was to observe the impact of liquid droplets on metal meshes that were bonded to a flat, solid plate. This represents a structured porous surface that can be characterized by two parameters, wire diameter and pore opening width, both of which were varied. Experiments were undertaken for different combination of drop radius, impact velocity, liquid surface tension and viscosity. Droplet impact was recorded using a high-speed video camera and the final state of the droplet observed. Several different outcomes were seen, including: a portion of the liquid penetrating into the pores; all of the liquid penetrating; and droplet splashing. Dimensionless maps were developed to predict conditions under which each outcome occurred. 


\section{Materials and methods}

The target surfaces were selected from a set of stainless steel metal meshes mainly used for filtration applications, with an equivalent pore sizes varying from 25 and $400 \mu \mathrm{m}$ (Plastok® Meshes and Filtration Ltd., Birkenhead, Merseyside, UK). Table 1 lists the properties of the meshes used in experiments. Fig. 1 shows scanning electron microscope (SEM) image of the meshes with the corresponding measured values of equilibrium and advancing contact angle. Note that these angles are measured macroscopically from the visible drop form, and they are not directly linked to the actual equilibrium contact angle between the fluid and the mesh material or to the microscopic contact angles closer to the liquid interface.

To avoid flexing of the meshes during droplet impact they were pressed on a flat stainless steel surface by means of a heavy steel ring.

Table 1 Mesh characteristics.

\begin{tabular}{cccc}
\hline Sample & Pore equivalent diameter $(\mu \mathrm{m})$ & $\begin{array}{c}\text { Wire diameter } \\
(\mu \mathrm{m})\end{array}$ & $\begin{array}{c}\text { Mesh thickness } \\
(\mu \mathrm{m})\end{array}$ \\
\hline 1 & 25 & 25 & 50 \\
2 & 50 & 36 & 72 \\
3 & 80 & 65 & 130 \\
4 & 100 & 100 & 200 \\
5 & 125 & 100 & 200 \\
6 & 150 & 125 & 250 \\
7 & 250 & 100 & 200 \\
8 & 400 & 220 & 440 \\
\hline
\end{tabular}

Experiments were conducted using three liquids: water, acetone and a glycerol-in-water solution composed by $20 \%$ of water and $80 \%$ of glycerol (by volume), to analyse the effect of viscosity and surface tension of liquid. The properties of the meshes and liquid properties are listed in Tables 1 and 2 respectively.

Table 2 Liquid properties

\begin{tabular}{ccrc}
\hline Liquid & $\begin{array}{c}\text { Density } \\
\left(\mathrm{kg} / \mathrm{m}^{3}\right)\end{array}$ & $\begin{array}{r}\text { Viscosity } \\
(\mathrm{mPa} \mathrm{s})\end{array}$ & $\begin{array}{c}\text { Surface } \\
\text { Tension }(\mathrm{N} / \mathrm{m})\end{array}$ \\
\hline Water & 996 & 1.00 & 0.073 \\
Acetone & 793 & 0.30 & 0.023 \\
$20 \%$ water $+80 \%$ Glycerol & 1118.6 & 10.00 & 0.067 \\
\hline
\end{tabular}


1)
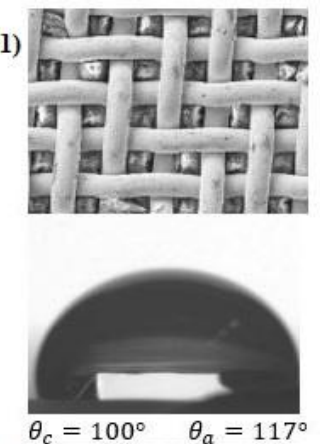

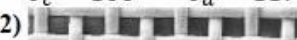
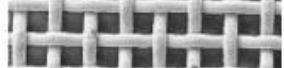

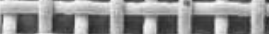

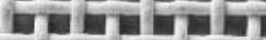

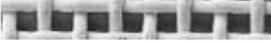

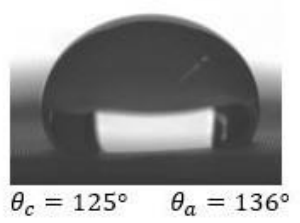

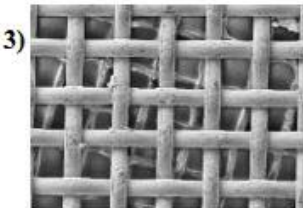
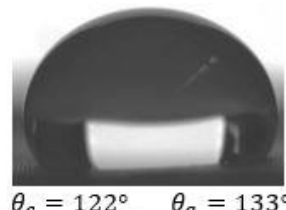

4)

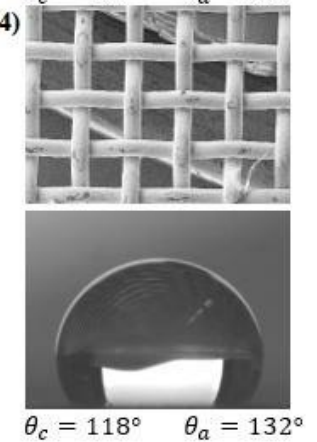

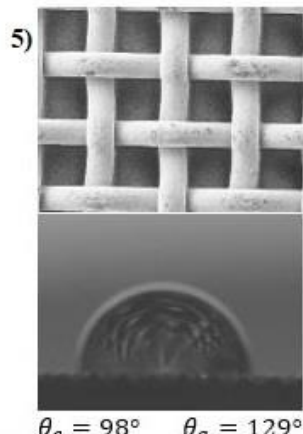
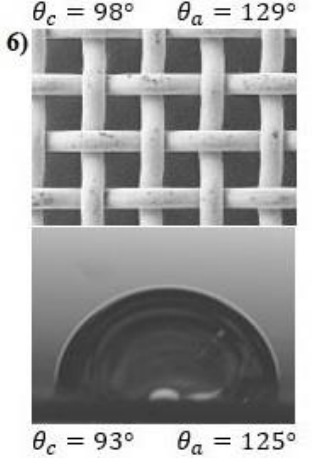

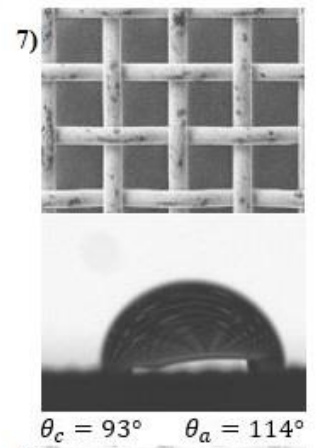

8)

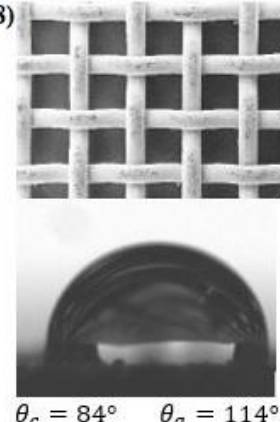

Fig. 1 SEM images of the stainless steel meshes. Equilibrium and advancing contact angles for water are also shown.

The droplet impact velocity was varied from $2 \mathrm{~m} / \mathrm{s}$ to $4 \mathrm{~m} / \mathrm{s}$ by adjusting the height of release of the droplet above the mesh between $20 \mathrm{~cm}$ and $80 \mathrm{~cm}$ and letting the droplet fall under the influence of gravity. Two needle sizes, 21 gauge ( $0.82 \mathrm{~mm}$ outer diameter, $0.51 \mathrm{~mm}$ inner diameter $)$ and $26 \mathrm{~s}$ gauge $(0.47 \mathrm{~mm}$ outer diameter, $0.13 \mathrm{~mm}$ inner diameter $)$ were used to produce drops with different diameters.

Droplet impact was photographed using a Photron Fastcam SA4 high speed camera (with a resolution of 1024x800 pixels), angled at $60^{\circ}$ to the horizontal plane. The test area was illuminated using a custom-built high-speed LED light source, synchronised to the high-speed camera. Fig. 2 shows a schematic of the experimental set-up used.

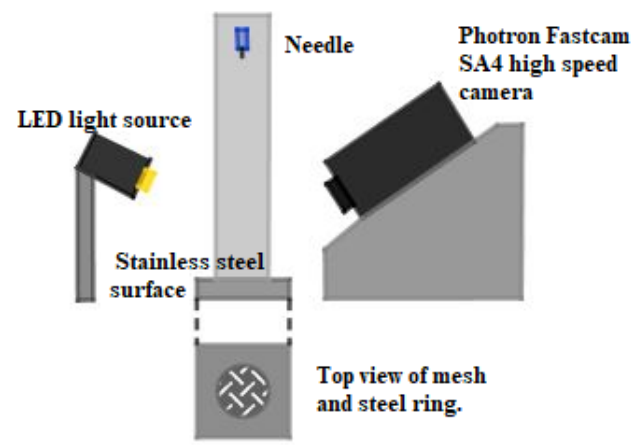

Fig. 2 Optical set up

Image analysis, using a MATLAB code, was used to measure the maximum spreading area of the droplet and the droplet initial diameter. The standard deviation for measurements of droplet diameter was $0.11 \mathrm{~mm}$ and for impact velocity was $0.14 \mathrm{~m} / \mathrm{s}$.

\section{Impact regimes}

A droplet landing on a mesh may produce 6 different outcomes that were observed and identified from photographs. Low velocity impacts result in deposition, partial imbibition or penetration. Higher velocity impacts lead to droplet 
splashing followed by deposition, partial imbibition and penetration. These different outcomes obtained from experiments are shown in the image sequences of Fig. 3.

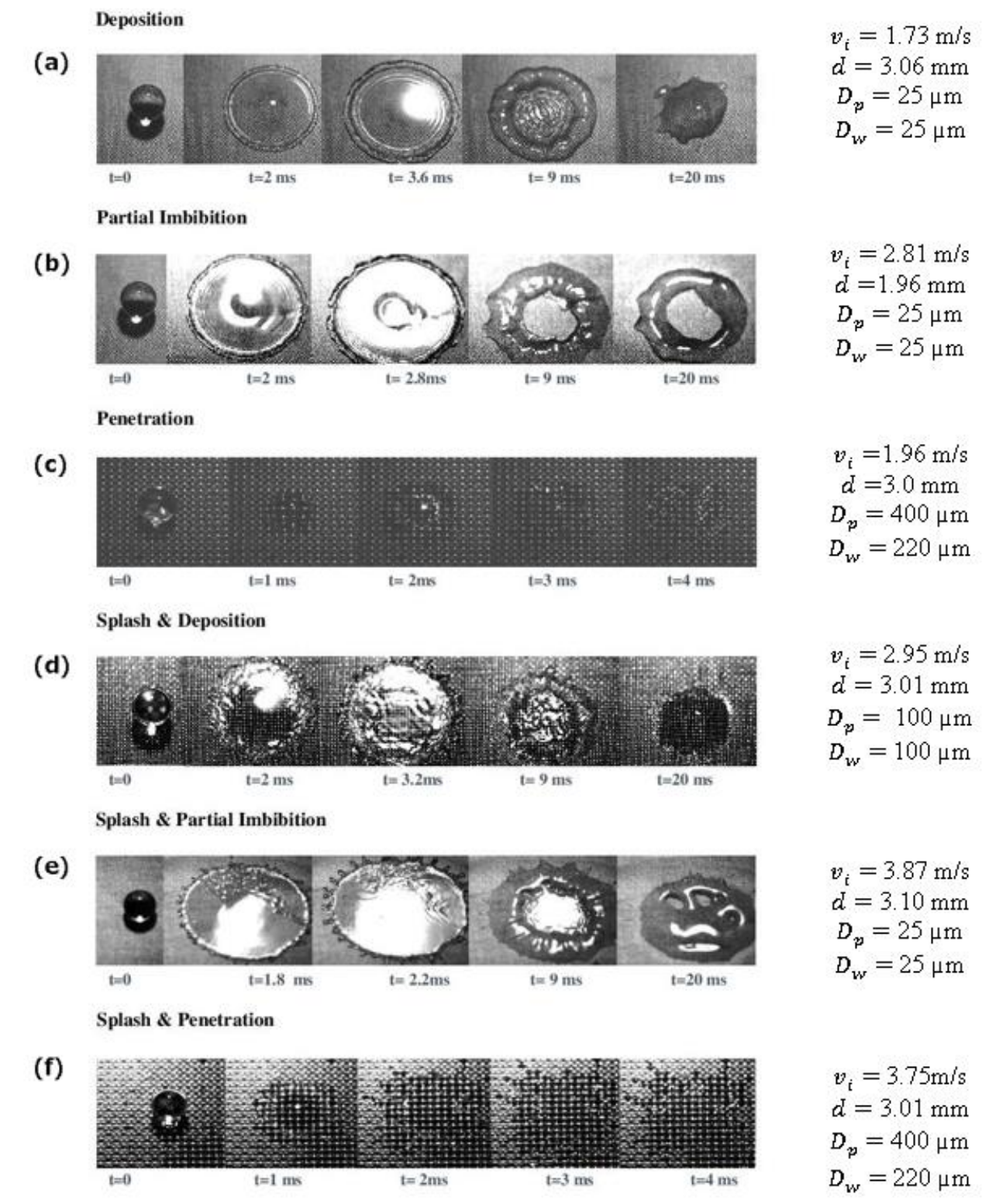

Fig. 3 Impact outcomes for water droplets. (a) Deposition: The droplet impacts on the substrate and after the spreading and recoiling the liquid remains deposited over the surface with no penetration. (b) Partial imbibition: The droplet impacts on the substrate. During the spreading and the recoiling part of the liquid penetrates below the surface. (c) Penetration: The droplet impacts on the substrates. Due to the larger dimension of the pores, no spreading occurs and the complete penetration process is almost instantaneous. (d) Splash \& Deposition: The droplet impacts on the surface with a higher velocity, after the splash all the liquid remains deposited on the surface. (e) Splash \& partial imbibition: The droplet impacts on the surface with a higher velocity, after the splash, part of the liquid penetrates below the surface. (f) Splash \& penetration: The droplet impacts on the surface with a higher velocity and due to the larger dimension of the pore, after the splash all the liquid penetrates below the surface.

In Fig. $3 a$, the droplet lands on the mesh $(t=0)$, spreads to its maximum extent in a circular shape ( $t=3.6 \mathrm{~ms})$, recoils $(\mathrm{t}=9$ $\mathrm{ms}$ ) and then comes to its equilibrium position where it is resting on the surface without all the liquid penetrating into the pores of the mesh. Partial imbibition (Fig. 3b) is defined as a state in which the central portion of the liquid in the spreading drop penetrates into the mesh during recoil while the remainder is deposited on the substrate in the shape of a ring. In Fig. $3 b$ the droplet impacts on the surface at time $t=0$ and begins to spread. At $t=2.8 \mathrm{~ms}$ the droplet reaches its maximum spreading diameter and already shows a depression at its center, where the pressure was greatest, due to imbibition of 
liquid into the mesh. Liquid recoil begins at $\mathrm{t}=9 \mathrm{~ms}$, and by $\mathrm{t}=20 \mathrm{~ms}$ the droplet has reached its equilibrium position with a hollow in its center. Fig. 3c shows droplet penetration, where the liquid passes through the mesh pores immediately following impact $(\mathrm{t}=2-4 \mathrm{~ms})$ and it is not possible to observe droplet spreading or recoiling.

Increasing droplet velocity led to droplet splashing, defined as the detachment of satellite droplets around the droplet periphery, immediately after impact. In Fig. $3 \mathrm{~d}$, splashing can be seen at $\mathrm{t}=2 \mathrm{~ms}$, before the droplet reaches its maximum spreading diameter. The droplet then recoils ( $\mathrm{t}=7.2 \mathrm{~ms}$ ) until it reaches equilibrium at $\mathrm{t}=20 \mathrm{~ms}$ with liquid deposited on the surface of the mesh. Fig. 3e shows a similar impact, except that at in the final state portions of the droplet have penetrated into the pores. Finally, in Fig. 3f, the liquid splashes after impact and then penetrates completely through the mesh pores ( $\mathrm{t}=2$ to $4 \mathrm{~ms}$ ) without showing any recoil.

\section{Results and discussion}

Penetration, which occurs immediately after droplet impact, occurs mainly when impact velocity is high (creating large inertial forces) or pore dimensions are large. Partial imbibition occurs at later time, during droplet recoiling, and is driven by capillary forces. Since the mechanisms that produce these two outcomes are different, and have different time scales, it is easier to examine them separately. Fig. 4 shows the different outcomes observed following the impact of water droplets as a function of impact velocity $\left(V_{i}\right)$ and pore diameter $\left(D_{p}\right)$. Fig. 4a shows the transition from deposition to partial imbibition, while Fig. $6 \mathrm{~b}$ shows the transition from deposition to penetration.
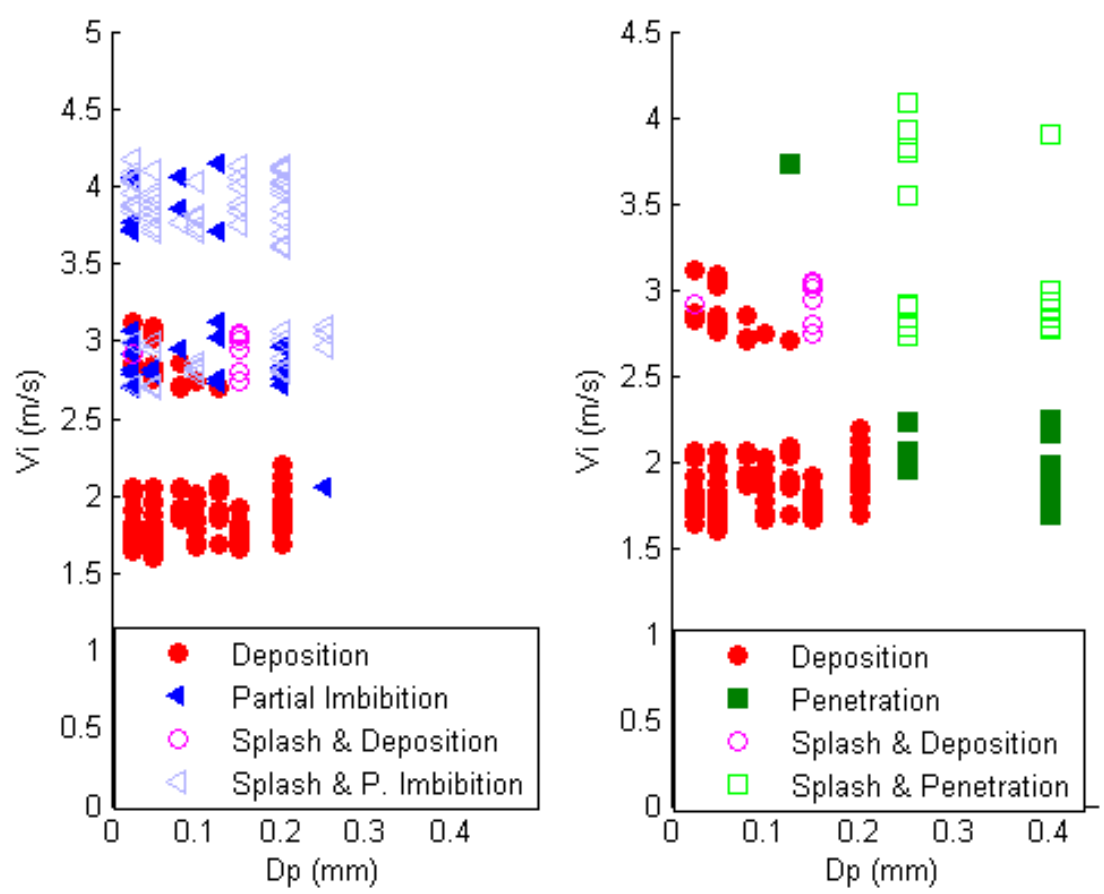

Fig. 4 (a) Regime distribution for water as a function of impact velocity $\left(v_{i}\right)$ and pore diameter $\left(D_{p}\right)$ for deposition and partial imbibition. (b) Regime distribution for water as a function of impact velocity $\left(v_{i}\right)$ and pore diameter $\left(D_{p}\right)$ for deposition and penetration.

Increasing the impact velocity above $3.5 \mathrm{~m} / \mathrm{s}$ results in partial imbibition (Fig. 4a). Making pore size larger than $0.1 \mathrm{~mm}$ leads first to partial imbibition (Fig. 4a), and then, when the pores are made even larger, to penetration (Fig. 4b). A combination of large pore size and high impact velocity produces both splashing and penetration (Fig. 4b). Similar experiments with acetone, which has much lower surface tension than water, usually led to total imbibition with deposition almost never seen. Impact of water-glycerol solution droplets, which have a much higher viscosity than water, typically resulted in deposition with penetration never occurring. Partial imbibition occurred only for the highest values of impact velocity.

Ryu et al. (2017) studied the penetration dynamics of water droplets through a mesh and expalined the transition from deposition and penetration by comparing the pressure driving penetratrion to the resisting capillary pressure. Looking at our results, this outcome can be identified as the partial imbibition in Fig. $3 \mathrm{~b}$ in which increasing the impact velocity, 
during the spreading and the recoling part of the liquids penetrates below the surface. The balance between the pressure driving penetration to the resisting capillary pressure can be written as

$C_{0} \rho v_{i}^{2}=p_{c}$

with $C_{0}$ being a proportional constant. From this balance they obtained the equation to find the velocity threshold for which imbibition occurs $\left(C_{0}=2.87\right)$.

$v_{c, i}=\frac{\sqrt{p_{c}}}{\sqrt{C_{0} \rho}}$

This equation agrees to a certain extent with our results, but deviates at low capillary pressures (Fig. 5)

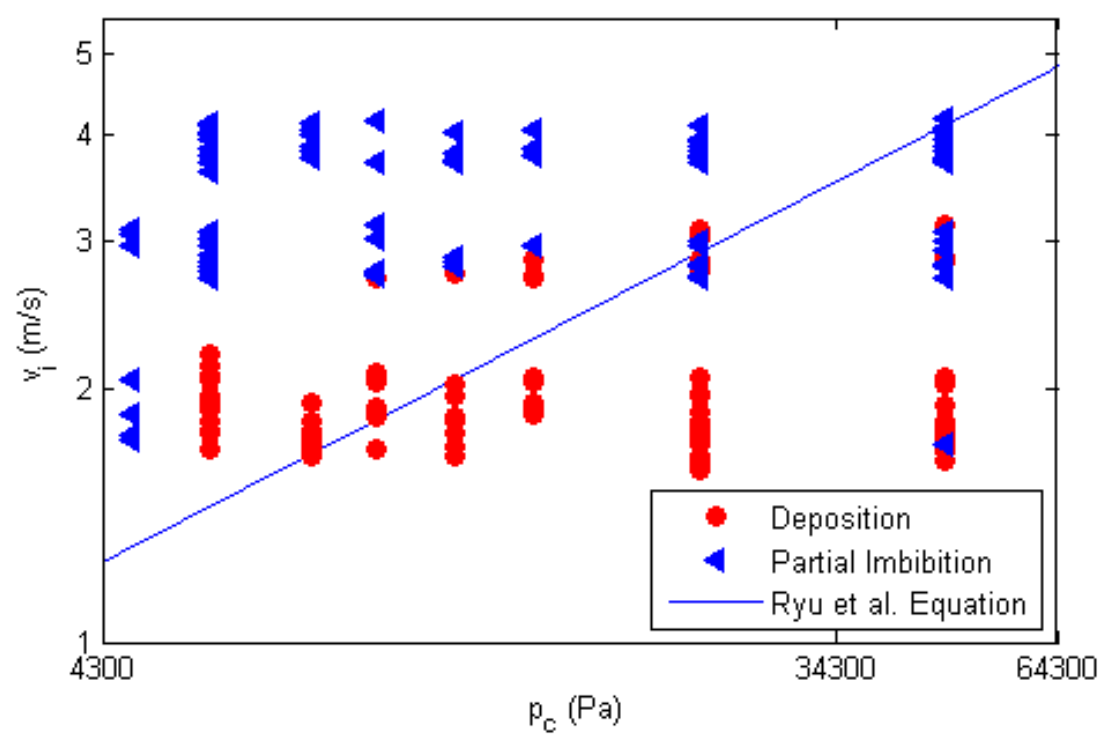

Fig. 5 Threshold velocity for deposition-imbibition (for water): comparison with Ryu et al. (2017)

Sahu et al. (2015) analysed the impact of a nanoparticle suspension into porous filter membranes and concluded that penetration of liquid into a porous medium is possible when the dynamic pressure is much higher than the capillary pressure, but can also occur when hydrodynamic focusing, which occurs when the drop diameter is much larger than the pore diameter, is observed. As Sahu et al. (2015) observed, and our results in Fig. 5 also show, penetration will occur at large values of dynamic pressure (large impact velocity) with respect to the capillary pressure, $p_{d} \gg p_{c}$, but will also be influenced by hydrodynamic focusing. To note, in our experiments the dimension of the droplet diameter is much larger than the pore diameter, $d \gg D_{p}$.

Lorenceau and Quéré (2003) studied the impact of a droplet on a surface pierced by a single hole, with diameters ranging from $0.13-0.45 \mathrm{~mm}$, and identified a critical speed above which the droplet is not entirely captured by the plate but passes through it after impact. Their analysis compared the stagnation pressure in the droplet after impact to the capillary pressure and shear force opposing the movement of liquid through the pore. Error! Reference source not found. shows the graph pointing at the threshold velocity limit for which the droplet is captured by the surface or ejected. The dashed line representing the velocity threshold for droplet capture is given by the equation

$$
R e=\frac{5.1 W e}{W e-3.6}
$$

It is possible to compare this result to our experimental data, considering the regimes of deposition and partial imbibition (Fig. 6). Lorenceau and Quéré (2003) used impact velocities sufficient to force part of the liquid droplet through the hole in a plate, but not high enough for complete penetration or splashing of the droplet. In defining the threshold for which the liquid droplet is not entirely captured by the surface and partially penetrates through the holes, 
our results find a good agreement with the curve defined by Lorenceau and Quéré (2003), even if the agreement is less evident for high viscous regimes, i.e. for low Reynolds numbers.

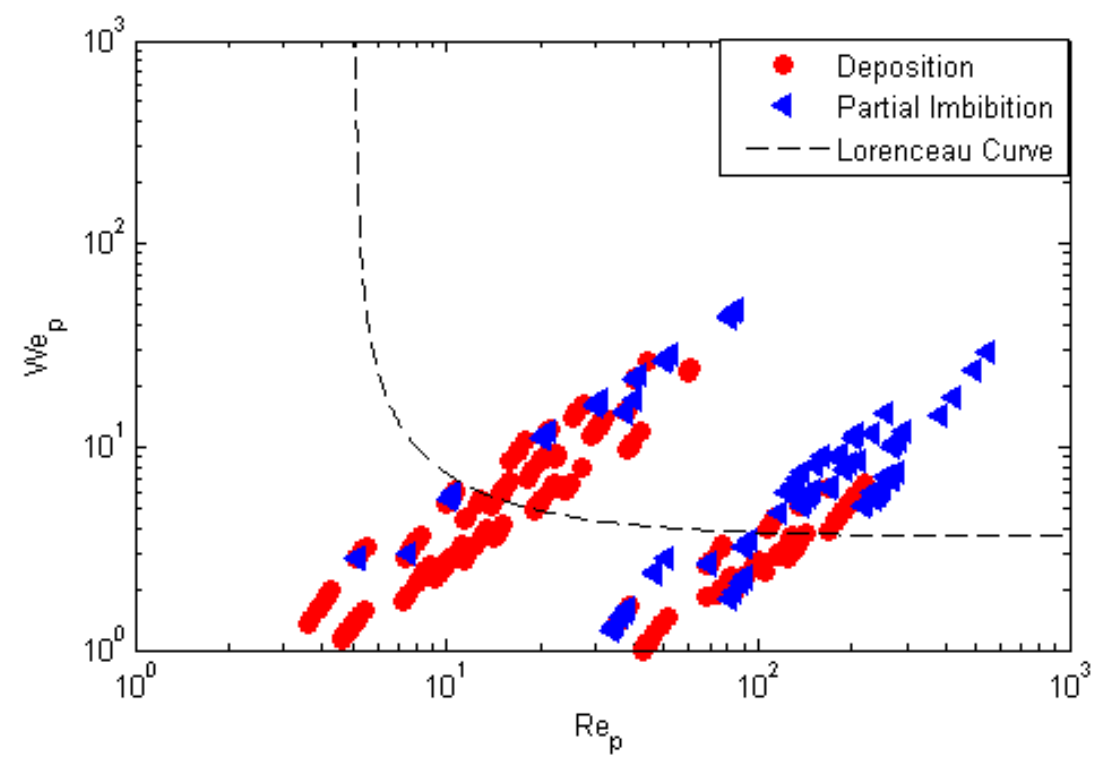

Fig. 6 Threshold velocity for capture: comparison with our results Lorenceau and Quéré (2003)

Focusing again on our results which involve also the complete penetration phenomenon and higher impact velocities, it is possible to do another consideration. The stagnation pressure when a droplet impacts on a solid surface is given by

$P_{S}=\frac{1}{2} \rho v_{i}^{2}$

The capillary pressure resisting liquid penetration into a pore with opening size $D_{p}$ is

$P_{c}=\frac{4 \sigma}{D_{p}}$

While the viscous shear stress can be estimated as being of order of magnitude

$\tau \sim \mu \frac{v_{i}}{D_{p}}$

In the limit of low velocity $\left(v_{i} \rightarrow 0\right)$ viscous forces are negligible and capillary forces dominate. For liquid to penetrate a pore we require $P_{s} \gg P_{c}$, or,

$W e_{p}=\frac{\rho v_{i}^{2} D_{p}}{\sigma} \gg 8$

alternatively, if the pore diameter is small $\left(D_{p} \rightarrow 0\right)$, or liquid viscosity is large, viscous forces dominate. In that case we require for liquid penetration to occur that $P_{S} \gg \tau$, or

$R e_{p}=\frac{\rho V D_{p}}{\mu} \gg 2$

$R e_{p}$ and $W e_{p}$ are the Reynolds and Weber numbers respectively, based on the mesh pore diameter as a length scale. These represent the lower limits on the value of $R e_{p}$ and $W e_{p}$ required for liquid penetration to occur. Fig. 7 shows the values of $R e_{p}$ and $W e_{p}$, for the cases where either liquid deposition or penetration was observed. Based on our results, the limits of $W e_{p}>8$ and $R e_{p}>50$ demarcate well the boundary between deposition and penetration. 


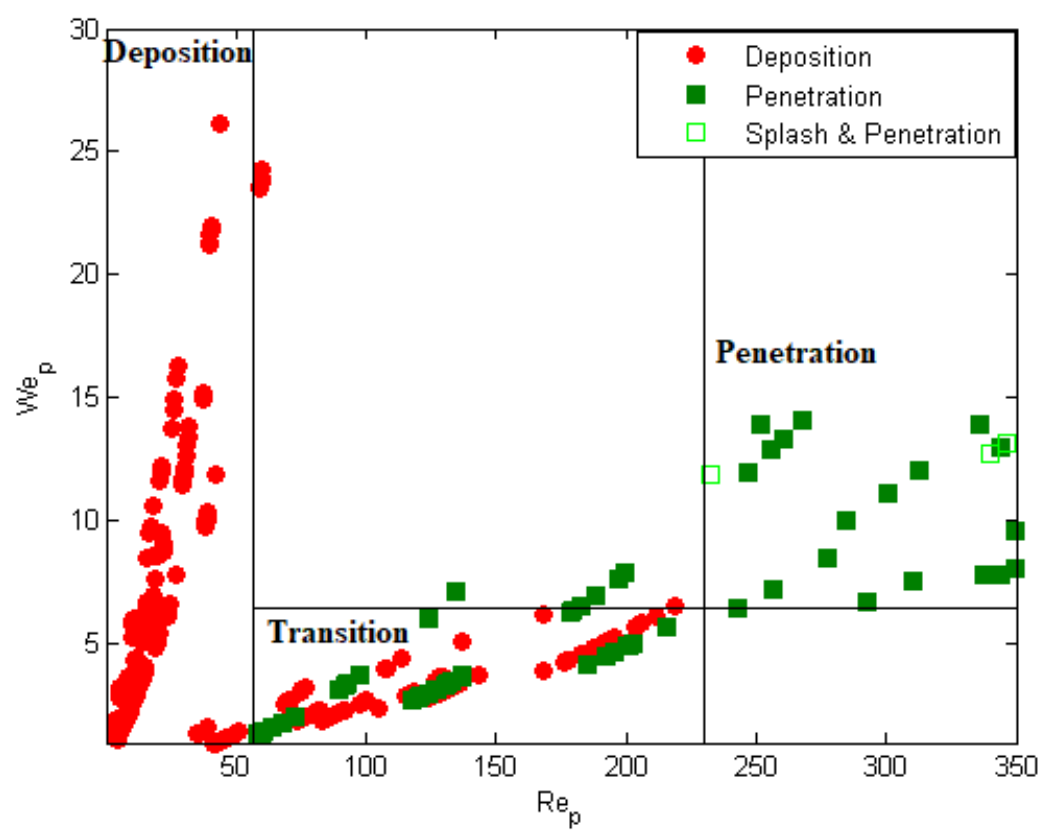

Fig. 7 Data distribution for water, acetone, water \& glycerol in function of $\mathrm{Re}_{p}$ and $\mathrm{We}_{p}$. Transition between deposition and penetration.

In the limit of small $R e_{p}$, the viscous shear forces dominate, preventing droplets from penetrating through the pores and resulting in droplet deposition on the mesh. At larger $R e_{p}\left(R e_{p}>50\right)$ viscous forces are no longer the dominant factor and penetration is controlled by a balance of capillary and inertial forces. For $50<R e_{p}<230$ and $W e_{p}<8$ a transition region occurs in which both capillary and inertial forces may affect penetration and penetration becomes the dominant outcome for $W e_{p}>8$. For $R e_{p}>230$, the only observable outcome is the penetration and inertial forces are dominant. Ryu et al. (2017) performed tests only using water droplets, while Lorenceau and Quéré (2003) worked with relatively low impact velocities, so that complete penetration into the mesh and splashing regime were not observed. For this reason, we find a different combination of parameters to define regime separation for a more general case.

The effect of the water hammer pressure, mentioned by Xu et al. (2017) and Brunet et al.( 2009), is not taken into account. The water hammer pressure effect, caused by the shockwave that propagates through the liquid once the droplet impinges on the surface, was not expected to have any significant influence on fluid flow considering the magnitude of the impact velocity (2-4 m/s) and the short time (a few nanoseconds) in which this effect takes place. Rioboo et al. (2001) observed that the first stage of droplet spreading following impact shows no ejection of a lamella from the body of the drop, though a shock wave should trigger lamella ejection. Considering the relatively long time (milliseconds) and low impact velocity of our experiments it was concluded that the dynamic pressure, rather than the water hammer pressure, plays a dominant role during impact.

To analyse the evolution of spreading of the droplet after the impact, measurements were made of droplet spreading diameters (d) as a function of time (t). The dimensionless diameter and time were defined as (Rioboo 2002):

$$
D^{*}=\frac{d(t)}{d_{o}} \quad, t^{*}=t \cdot \frac{v_{i}}{d 0}
$$

where $d_{o}$ is the droplet initial diameter, $d(t)$ the time-varying spreading diameter after impact and $v_{i}$ the impact velocity. Fig. 8 (a) shows the variation of spreading diameter (spread factor) with time for water droplets landing on a $25 \mu \mathrm{m}$ diameter mesh at three different impact velocities. The spread factor increases with impact velocity, as observed for droplet impact on a solid surface. Table 3 lists the values of We for each of these velocities. For a $25 \mu \mathrm{m}$ mesh $\mathrm{We}$ is always less than the critical value of 8 , implying that there is no penetration through the mesh. Droplet impact dynamics are similar to those seen on an impermeable surface. The maximum spread diameter of a droplet landing on a smooth surface is given by Pasandideh-Fard et al. (1996):

$$
D_{\max }=\frac{\sqrt{W e+12}}{3\left(1-\cos \theta_{a}\right)+4\left(\frac{W e}{\sqrt{R e}}\right)}
$$


where $\theta_{a}$ is the equilibrium advenging contact angle after the spread and $D_{\max }$ the maximum spreading diameter. For the cases of a water droplet with $3 \mathrm{~mm}$ diameter and three impact velocities of 2,3 and $4 \mathrm{~m} / \mathrm{s}$, the maximum spreading diameter on a smooth stainless steel surface from eq. (10) would be respectively 4, 4.6 and 5. The measured spread factors are approximately $25 \%$ lower than these predictions: the difference is due to the effect of the wires and the topology of the mesh that create barriers to the outward flow, effectively creating a sort of surface roughness. Due to the tilted angle of the camera optics, it is possible to trace the spreading evolution just up to $6 \mathrm{~ms}$ after the impact.

(a)

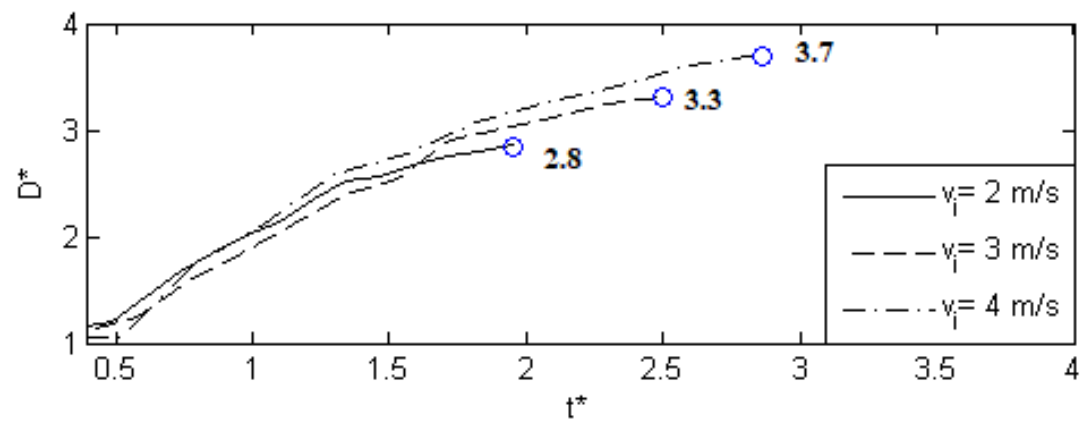

(b)

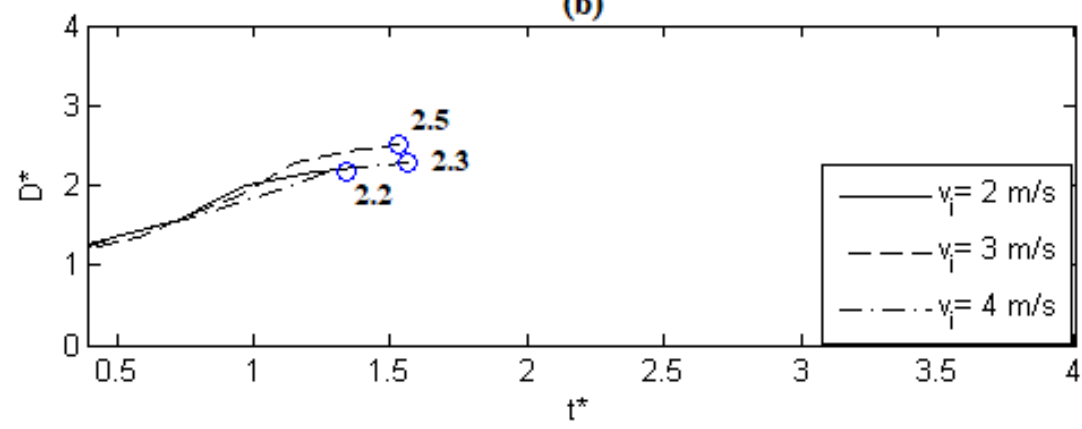

Fig. 8 Evolution of dimensionless spreading diameter with time for water droplets with $d=3 \mathrm{~mm}$. (a) $D_{p}=25 \mu \mathrm{m}, D_{w}=25$ $\mu \mathrm{m}$, (b) $D_{p}=200 \mu \mathrm{m}, D_{w}=125 \mu \mathrm{m}$.

Fig. 8(b) shows the evolution of droplet diameter during impact on a mesh with $200 \mu \mathrm{m}$ pores. The standard deviation on the maximum spreading for all the cases is about $4 \%$. At the lowest impact velocity $(2 \mathrm{~m} / \mathrm{s})$, the maximum spread factor is 2.2 , lower than it was on a the $25 \mu \mathrm{m}$ mesh, which corresponds to $\mathrm{D}^{*}=2.8$. The reduced spread can be explained by two different reasons: (i) for a larger pore size, a higher percentage of liquid will penetrate below the surface leading to a lower spreading diameter; (ii) larger pore size corresponds also a larger wire diameter and consequently during spreading, the greater "roughness" will enhance the viscous dissipation leading to a smaller spreading size. The $200 \mu \mathrm{m}$ pore size mesh has a wire diameter of $125 \mu \mathrm{m}$, whereas the $25 \mu \mathrm{m}$ pore size mesh has a wire diameter of only $25 \mu \mathrm{m}$. At the lowest impact velocity on a mesh with $200 \mu \mathrm{m}$ pores, $\mathrm{We}_{\mathrm{p}}=9.1$, which is barely above the limit for penetration to occur. It is likely that the reduced droplet spreading in this case is the result of surface roughness due to the larger wire diameter rather than liquid being trapped in pores. When impact velocity is increased to $3 \mathrm{~m} / \mathrm{s}$ the droplet spread increases, though it is less than it was on a $25 \mu \mathrm{m}$ mesh for the same impact velocity (compare Fig. 8a and Fig. 8b). Finally, at the highest impact velocity, $4 \mathrm{~m} / \mathrm{s}$, the maximum spread was slightly less than it was for the $3 \mathrm{~m} / \mathrm{s}$ impact. The Weber number We $=$ 22.5 for $\mathrm{v}_{\mathrm{i}}=3 \mathrm{~m} / \mathrm{s}$ and $\mathrm{We}_{\mathrm{p}}=41.5$ for $\mathrm{v}_{\mathrm{i}}=4 \mathrm{~m} / \mathrm{s}$, implying significant penetration of liquid into pores for both cases. Increased loss of liquid in the pores would explain why a smaller amount of liquid was left on the surface to spread.

To determine if the volume of pores was sufficient to cause a significant loss of liquid, volume of the voids in the mesh under the droplet at its maximum spread was calculated. Consider a droplet that has spread to its maximum extent, the area covered by the spreading droplet is given by

$$
A_{s p}=\frac{D \max ^{2}}{4} \pi
$$


To estimate the number of pores (x) under the droplet it was assumed that there is a square of equal area with each side covering $\mathrm{x}$ pores and $\mathrm{x}+1$ wires so that

$$
A_{s p}=A_{e q}=\left(D_{p} \cdot x+(x+1) D_{w}\right)^{2}
$$

Consequently, the total number of pores per side will be

$$
x=\operatorname{int}\left[\frac{\left(\sqrt{A_{s p}}-D_{w}\right)}{D_{p}+D_{w}}\right]
$$

The void volume will be given by multiplying the void volume of a single pore by the total number of covered pores at the maximum spreading.

Considering that the mesh consists of overlapped wires, the volume of the single pore will be given by

$$
V_{p}=2 D_{w} \cdot\left(D_{p}+D_{w}\right)^{2}-2 \cdot\left(D_{p}+D_{w}\right) \cdot \frac{\pi \cdot D_{w}^{2}}{4}
$$

Consequently, the entire void volume is

$$
V_{\text {void }}=x^{2} \cdot V_{p}
$$

Table 3 lists the void volume as a percentage of the liquid volume in a drop for different impact conditions and mesh sizes. At the highest impact velocity $(4 \mathrm{~m} / \mathrm{s})$ on a mesh with $0.2 \mathrm{~mm}$ pores the void volume is very significant, being $38 \%$ of the drop volume. At a lower impact velocity the droplet spread is larger and therefore void volume is even greater, $48 \%$. However, since $\mathrm{We}_{\mathrm{p}}$ is lower less liquid would have entered the pores. The void volume measure gives an estimate of the percentage of liquid that penetrates the pores after the impact. Because the meshes were placed on a solid plate, it was not possible to directly observe the volume of liquid penetrating through the mesh.

Table 3 Void volume at the maximum spreading for water

\begin{tabular}{ccccccc}
\hline Experiment $\mathrm{N}$ & $v_{i}(\mathrm{~m} / \mathrm{s})$ & $\mathrm{We}_{\mathrm{p}}$ & $D_{\max }(\mathrm{mm})$ & $D_{w}(\mathrm{~mm})$ & $D_{p}(\mathrm{~mm})$ & Void volume $\%$ \\
\hline 1 & 2 & 1.1 & 8.6 & 0.025 & 0.025 & 12 \\
2 & 3 & 2.8 & 9.8 & 0.025 & 0.025 & 16 \\
3 & 4 & 5.2 & 10.8 & 0.025 & 0.025 & 20 \\
4 & 2 & 4.6 & 6.4 & 0.065 & 0.1 & 20 \\
5 & 3 & 11.2 & 7.7 & 0.065 & 0.1 & 29 \\
6 & 4 & 20.8 & 7.0 & 0.065 & 0.1 & 24 \\
7 & 2 & 9.1 & 6.2 & 0.125 & 0.2 & 36 \\
8 & 3 & 22.5 & 7.2 & 0.125 & 0.2 & 38 \\
9 & 4 & 41.5 & 6.4 & 0.125 & 0.2 & 38 \\
\hline
\end{tabular}

In case of penetration, it is not possible to define a spreading of the droplet on the surface because the entire droplet penetrates inside the pore after the first millisecond. Considering the instant in which the droplet touches the surface, the time required for the first half of the spherical droplet to penetrate inside the surface will be given by

$t_{p}=\frac{d}{2 v_{i}}$

Once that the first half of the droplet penetrates inside the surface, no spreading is detectable and penetration occurs. This leads to a dimensionless time equal to

$t_{p}^{*}=\frac{t_{p} v_{i}}{d}=\frac{1}{2}=0.5$

This result is in good agreement with the spreading analysis in Fig. 8, in which the spreading evolution takes part for dimensionless time larger than 0.5 . 
When $t^{*}>0.5>t_{p}$ The spreading of the droplet occurs and, depending on the pore diameter, a partial imbibition of the liquid inside the surface may occur,

When $t^{*}<0.5<t_{p}$

All the liquid may penetrate through the mesh without leading to any spreading.

Having a different time scale, it was chosen to define separately the transition between deposition and partial imbibition and the deposition and penetration in two regimes maps. In order to determine a transition map in term of dimensionless number if was chosen to modify the Reynolds and Weber number, using as characteristic length the diameter of the droplet.

$W e=\frac{\rho d v_{i}^{2}}{\sigma}$

$R e=\frac{\rho d v_{i}}{\mu}$

It was observed that the separation of the outcomes is not clearly defined without introducing a geometrical parameter given by the ratio of the empty area over the full area of the mesh pore

$\gamma=\frac{A_{\text {full }}}{A_{\text {empty }}}=\frac{\left(D_{p}+D_{w}\right)^{2}}{D_{P}^{2}}=\frac{D_{p}^{2}+2 D_{p} D_{w}+D_{w}^{2}}{D_{p}^{2}}=\frac{D_{p}^{2}\left(1+2 \frac{D_{w}}{D_{p}}+\frac{D_{w}^{2}}{D_{p}^{2}}\right)}{D_{p}^{2}}=D_{p}^{2} \cdot \frac{\left(1+\frac{D_{w}}{D_{p}}\right)^{2}}{D_{p}^{2}}=\left(1+\frac{D_{w}^{2}}{D_{p}^{2}}\right)$

In fact, considering the previous analysis it is clear that not only does the pore diameter play a role in the penetration of the liquid, but also the wire diameter which will influence the percentage of liquid penetration and the void volume. In case of partial imbibition, a higher thickness of the wire will lead to a higher liquid imbibition. In case of penetration, a higher dimension of the wire and a larger pore diameter will lead to the complete penetration of the liquid below the surface

\section{Case $\mathbf{t}^{*}<0.5=\mathbf{t}_{\mathrm{p}}^{*}$}

For dimensionless times lower than $t_{p}^{*}$, drop penetration may occur. The regimes map in Fig. 9 shows the separation between deposition and penetration. The Weber number alone results is insufficient to obtain a satisfactory description of outcome distribution. Therefore, the Weber has been coupled with the Re number, to capture the viscosity effects. The best choice of the parameter on y-axis was evaluated by:

$$
M=R e^{0.8} W e^{2.1}
$$

The exponentials in the equation were determined using a Matlab code that measured the distance between data points on a $M-\gamma$ plot, where each point was initially classified as lying in one of two different impact regimes, deposition or penetration. The code attempts to maximize the average distance between points belonging to different regimes while minimising the average distance between points in the same regime. Four matrices of distance were constructed, listing the distance of each data point in a particular domain from all the other points belonging to the same domain, and from all points of the other domain. From the matrices it was possible to determine average distance vectors. If a given data point was on average closer to points belonging to the other domain than those of the same domain, it was classified as an error, and the best exponential values were those that led to the smallest number of errors. Finally, the code analysed the distance between the orthocentres of the two domains to select exponentials that maximised the distance between the orthocentres and consequently led to better separation between the domains.

The transition with the penetration regime begins at $M>24$ until penetration becomes the only dominant outcome for $\mathrm{M}>34$. 


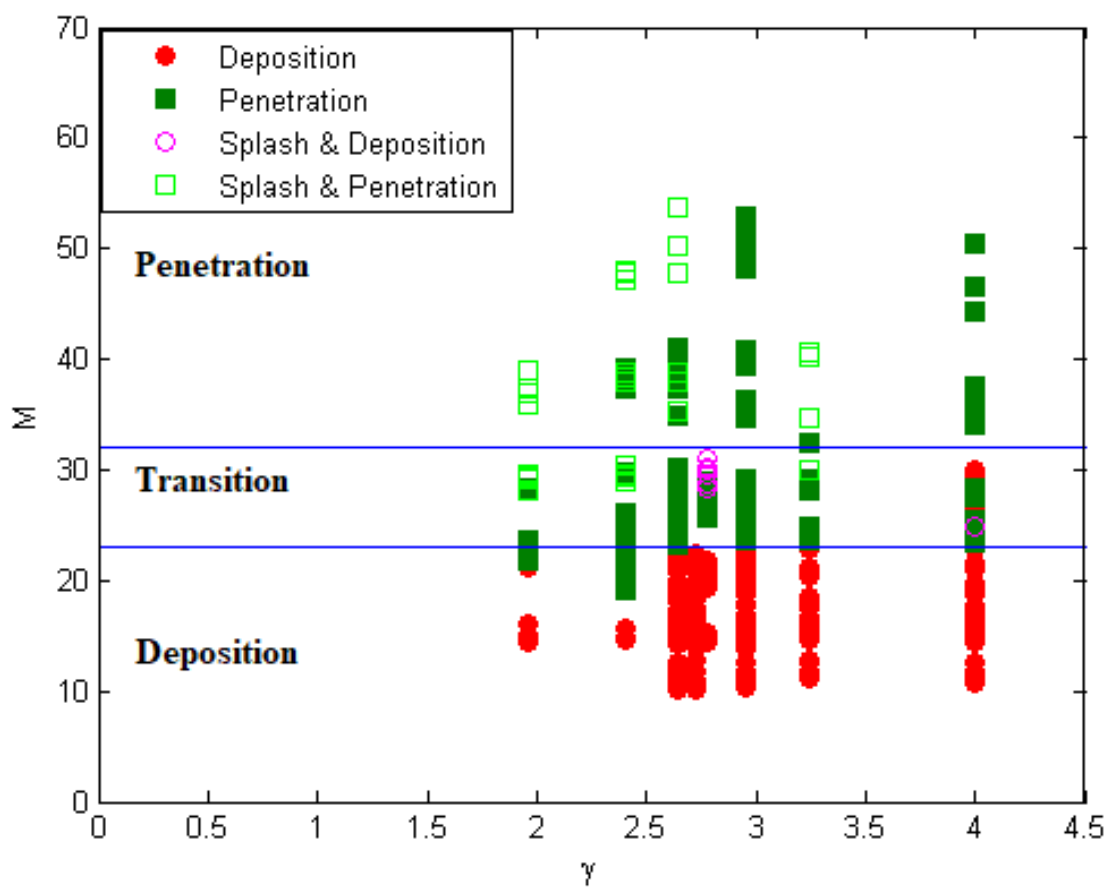

Fig. 9 Regime distribution for all the liquids as a function of $\gamma$ and $\mathrm{M}$, transition between deposition and penetration

\section{Case $t^{*}>t_{p}^{*}$}

For $t^{*}>0.5$, it is possible to observe the spreading of the droplet on the surface and consequently the complete penetration does not occur.

In Fig. 10, the viscosity plays a major role in describing the deposition-partial imbibition transition and therefore the best parameter on the y-axis is exclusively given by the Reynolds number. Increasing the viscosity and for lower Reynolds numbers, the dominant outcome is given by the deposition. The transition between deposition and partial imbibition which starts for Re>500, will occur for a higher velocity or a lower viscosity. Higher values of Re $(\mathrm{Re}>10000)$ also relate to a splash outcome. 


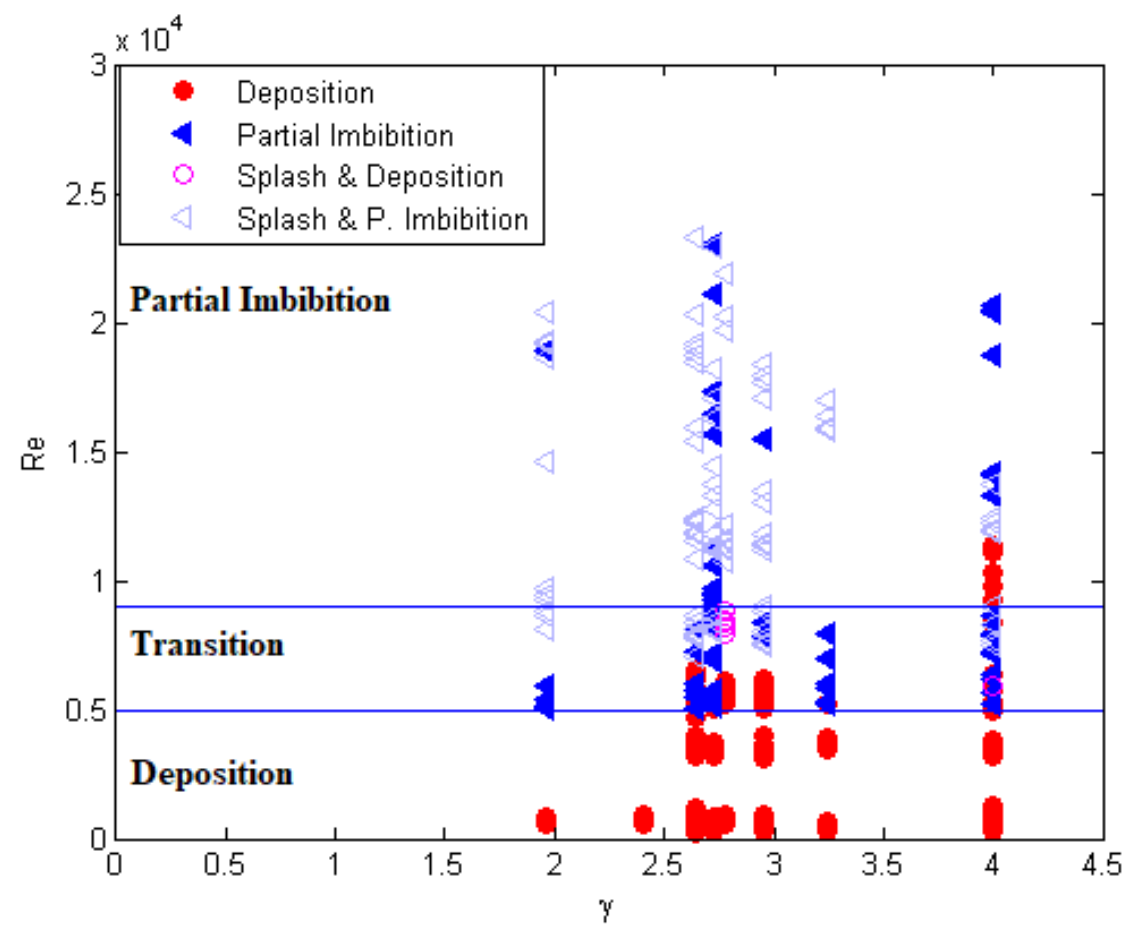

Fig. 10 Regime distribution for all the liquids as a function of $\gamma$ and Re transition between deposition and partial imbibition

\section{Conclusions}

Droplet impact on metallic meshes with a wide range of pore sizes was observed. Several different outcomes were identified, including deposition, penetration, imbibition and splashing. Penetration of droplets through the mesh is enhanced by increasing the ratio of dynamic pressure with respect to the capillary pressure $\left(p_{d} \gg p_{c}\right)$, and it is also influenced by hydrodynamic focusing which occurs when the of the droplet diameter is much larger than the pore diameter $\left(d \gg D_{p}\right)$. It was found that it was not possible to represent the different outcome regimes without including a geometrical parameter that included the wire mesh diameter. To achieve a clear distinction between different impact regimes, it is necessary to use dimensionless numbers that account for the liquid properties, including density, viscosity and surface tension. To be able to predict a wide range of outcomes which include complete penetration of the droplet into pores and splashing, maps of two different regimes were proposed. The initial phase, $t *<0.5$, defines the separation between deposition and the complete penetration of the droplet inside the surface. For $t *>0.5$, a second regime map is proposed, defining the transition between deposition and partial imbibition. In both cases it was observed that a higher impact velocity leads also splashing. Two new dimensionless parameters, $M$ and $\gamma$, are introduced, and the outcomes of the impact are therefore predicted for the given range of experimental parameters. This work allows to predict the outcome of droplet impact on a mesh membrane at different time scales, considering liquid having different physical properties and meshes with a wide range of pores size.

\section{References}

Bertola V (2008) Some Applications of Controlled Drop Deposition on Solid Surfaces. Recent Patents Mech Eng 1:167-174. doi: 10.2174/1874477X10801030167

Brunet P, Lapierre F, Zoueshtiagh F, et al (2009) To grate a liquid into tiny droplets by its impact on a hydrophobic microgrid. 94-96. doi: 10.1063/1.3275709

Chandra S, Avedisian CT (1992) Observations of droplet impingement on a ceramic porous surface. Int J Heat Mass Transf 35:2377-2388. doi: 10.1016/0017-9310(92)90080-C

Choi M, Son G, Shim W (2017) A level-set method for droplet impact and penetration into a porous medium. Comput Fluids 145:153-166. doi: 10.1016/j.compfluid.2016.12.014

Clarke A, Blake TD, Carruthers K, Woodward A (2002) Surfaces. 2980-2984

Delbos A, Lorenceau E, Pitois O (2010) Forced impregnation of a capillary tube with drop impact. J Colloid Interface 
Sci 341:171-177. doi: 10.1016/j.jcis.2009.09.016

Gambaryan-Roisman T (2014) Liquids on porous layers: Wetting, imbibition and transport processes. Curr Opin Colloid Interface Sci 19:320-335. doi: 10.1016/j.cocis.2014.09.001

He P, Liu Y, Qiao R (2015) Fluid dynamics of the droplet impact processes in cell printing. Microfluid Nanofluidics 18:569-585. doi: 10.1007/s10404-014-1470-3

Hicks PD, Purvis R (2017) Gas-cushioned droplet impacts with a thin layer of porous media. J Eng Math 102:65-87. doi: 10.1007/s10665-015-9821-y

Josserand C, Thoroddsen ST (2016) Drop Impact on a Solid Surface. Annu Rev Fluid Mech 48:365-391. doi: 10.1146/annurev-fluid-122414-034401

Karapetsas G, Chamakos NT, Papathanasiou AG (2016) Efficient modelling of droplet dynamics on complex surfaces. J Phys Condens Matter 28:. doi: 10.1088/0953-8984/28/8/085101

Kim WS, Lee SY (2014) Behavior of a water drop impinging on heated porous surfaces. Exp Therm Fluid Sci 55:6270. doi: 10.1016/j.expthermflusci.2014.02.023

Kumar SM, Deshpande AP (2006) Dynamics of drop spreading on fibrous porous media. Colloids Surfaces A Physicochem Eng Asp 277:157-163. doi: 10.1016/j.colsurfa.2005.11.056

Lee JB, Radu AI, Vontobel P, et al (2016) Absorption of impinging water droplet in porous stones. J Colloid Interface Sci 471:59-70. doi: 10.1016/j.jcis.2016.03.002

Letters PR (2017) Water Penetration through a Superhydrophobic Mesh During a Drop Impact. 014501:1-5. doi: 10.1103/PhysRevLett.118.014501

Liu Y, Wang Z (2016) Superhydrophobic porous networks for enhanced droplet shedding. Sci Rep 6:1-7. doi: $10.1038 /$ srep33817

Lorenceau É, Quéré D (2003) Drops impacting a sieve. J Colloid Interface Sci 263:244-249. doi: 10.1016/S00219797(03)00126-7

Mazloomi Moqaddam A, Chikatamarla SS, Karlin I V. (2017) Drops bouncing off macro-Textured superhydrophobic surfaces

Pasandideh-Fard M, Qiao YM, Chandra S, Mostaghimi J (1996) Capillary effects during droplet impact on a solid surface. Phys Fluids 8:

Pittoni PG, Lin Y, Lin S (2014) Applied Surface Science The impalement of water drops impinging onto hydrophobic / superhydrophobic graphite surfaces : the role of dynamic pressure, hammer pressure and liquid penetration time. Appl Surf Sci 301:515-524. doi: 10.1016/j.apsusc.2014.02.115

Reis NC, Griffiths RF, Santos JM (2004) Numerical simulation of the impact of liquid droplets on porous surfaces. J Comput Phys 198:747-770. doi: 10.1016/j.jcp.2004.01.024

Rioboo R, Tropea C, Marengo M (2001) Outcomes From a Drop Impact on Solid Surfaces. At Sprays 11:12. doi: 10.1615/AtomizSpr.v11.i2.40

Roisman I V., Lembach A, Tropea C (2015) Drop splashing induced by target roughness and porosity: The size plays no role. Adv Colloid Interface Sci 222:615-621. doi: 10.1016/j.cis.2015.02.004

Romdhani Z, Baffoun A, Hamdaoui M, Roudesli S (2014) Drop impact on textile material: Effect of fabric properties. Autex Res J 14:145-151. doi: 10.2478/aut-2014-0010

Sahu RP, Sett S, Yarin AL, Pourdeyhimi B (2015) Impact of aqueous suspension drops onto non-wettable porous membranes: Hydrodynamic focusing and penetration of nanoparticles. Colloids Surfaces A Physicochem Eng Asp 467:31-45. doi: 10.1016/j.colsurfa.2014.11.023

Weclas M (2010) Potential of Porous-Media Combustion Technology as Applied to Internal Combustion Engines. J Thermodyn 2010:1-39. doi: 10.1155/2010/789262

Xu J, Xie J, He X, et al (2017) Water drop impacts on a single-layer of mesh screen membrane: Effect of water hammer pressure and advancing contact angles. Exp Therm Fluid Sci 82:83-93. doi: 10.1016/j.expthermflusci.2016.11.006

Yamamoto K, Takezawa H, Ogata S (2016) Droplet impact on textured surfaces composed of commercial stainless razor blades. Colloids Surfaces A Physicochem Eng Asp 506:363-370. doi: 10.1016/j.colsurfa.2016.06.024

Yarin AL (2006) DROP IMPACT DYNAMICS: Splashing, Spreading, Receding, Bouncing.... Annu Rev Fluid Mech 38:159-192. doi: 10.1146/annurev.fluid.38.050304.092144

Zhang G, Quetzeri-Santiago MA, Stone CA, et al (2018) Droplet impact dynamics on textiles. Soft Matter. doi: 10.1039/C8SM01082J

Zhang R, Farokhirad S, Lee T, Koplik J (2014) Multiscale liquid drop impact on wettable and textured surfaces. Phys Fluids 26:. doi: 10.1063/1.4892083 